\title{
Global fits to neutrino oscillation data*
}

\author{
Thomas Schwett \\ Scuola Internazionale Superiore di Studi Avanzati, via Beirut 2-4, 34014 Trieste, Italy
}

\begin{abstract}
I summarize the determination of neutrino oscillation parameters within the three-flavor framework from world neutrino oscillation data with date of May 2006, including the first results from the MINOS long-baseline experiment. It is illustrated how the determination of the leading "solar" and "atmospheric" parameters, as well as the bound on $\theta_{13}$ emerge from an interplay of various complementary data sets. Furthermore, I discuss possible implications of sub-leading three-flavor effects in present atmospheric neutrino data induced by $\Delta m_{21}^{2}$ and $\theta_{13}$ for the bound on $\theta_{13}$ and non-maximal values of $\theta_{23}$, emphasizing, however, that these effects are not statistically significant at present. Finally, in view of the upcoming MiniBooNE results I briefly comment on the problem to reconcile the LSND signal.
\end{abstract}

\section{INTRODUCTION}

In the last ten years or so we have witnessed huge progress in neutrino oscillation physics. The outstanding experimental results lead to quite a clear overall picture of the neutrino sector. We know that there are two mass-squared differences separated roughly by a factor of 30, and in the lepton mixing matrix there are two large mixing angles, and one mixing angle which has to be small. In this talk I review the present status of neutrino oscillations by reporting the results of a global analysis of latest world neutrino oscillation data from solar 1, 2, 3], atmospheric [4, 5], reactor 6, 7], and accelerator [8, 9] experiments, including the recent data from the MINOS long-baseline experiment [9]. This analysis is performed in the three-flavor framework and is based on the work published in Refs. 10, 11] (see also the hep-ph archive version 5 of Ref. [11] for updated results). In Sec. III I discuss the determination of the leading "solar" and "atmospheric" parameters, whereas Sec. III deals with the bound on $\theta_{13}$ from global data. The status of three-flavor oscillation parameters is summarized in Tab. I In Sec. IV a discussion of sub-leading effects in atmospheric data is given, and in Sec. $\nabla] \mathrm{I}$ comment on attempts to reconcile the result of the LSND experiment [12] with the global oscillation data.

\section{LEADING OSCILLATION PARAMETERS}

In this section I discuss the determination of the leading oscillation parameters, the "solar" parameters $\theta_{12}, \Delta m_{21}^{2}$, and the "atmospheric" parameters $\theta_{23}, \Delta m_{31}^{2}$. In both cases we have an independent confirmation of neutrino oscillations from very different experiments, and the final allowed regions

*Talk presented at SNOW2006, Stockholm, 2-6 May 2006

$\dagger$ Electronic address: schwetz@sissa.it for the oscillation parameters emerge from an interplay of complementary data: The determination of the mixing angle is dominated by experiments with natural neutrino sources (solar and atmospheric neutrinos), whereas the mass-squared differences are more accurately determined by man-made neutrinos (from reactors and accelerators). This complementarity is illustrated in Fig. 1].

Details of our solar neutrino analysis can be found in Ref. 10] and references therein. We use data from the Homestake, SAGE, GNO, and SK experiments [1], and the SNO day-night spectra from the pure $\mathrm{D}_{2} \mathrm{O}$ phase 2], but the $\mathrm{CC}, \mathrm{NC}$, and ES rates from the SNO salt-phase are updated according to the latest 2005 data [3]. The predictions for the solar neutrino fluxes are taken from Ref. [13]. For the KamLAND analysis we are using the data from Ref. [6] equally binned in $1 / E_{\mathrm{pr}}\left(E_{\mathrm{pr}}\right.$ is the prompt energy deposited by the positron), and we include earth matter effects and flux uncertainties following Ref. [14] (see the appendix of Ref. 11] for further details).

We observe from Fig. 1 (left) a beautiful agreement of solar and KamLAND data. Moreover, the complementarity of the two data sets allows a rather precise determination of the oscillation parameters: The evidence of spectral distortion in KamLAND data provides a strong constraint on $\Delta m_{21}^{2}$, and leads to the remarkable precision of $4 \%$ at $1 \sigma$ (compare Tab. (1). Alternative solutions around $\Delta m_{21}^{2} \sim$ $2 \times 10^{-4} \mathrm{eV}^{2}\left(\sim 1.4 \times 10^{-5} \mathrm{eV}^{2}\right)$, which are still present in the KamLAND-only analysis at 99\% C.L., are ruled out from the combined KamLAND+solar analysis at about $4 \sigma(5 \sigma)$. In contrast to $\Delta m_{21}^{2}$, the determination of the mixing angle is dominated by solar data. Especially recent results from the SNO experiment provide a strong upper bound on $\sin ^{2} \theta_{12}$, excluding maximal mixing at more than $5 \sigma$.

Oscillations with the "atmospheric" parameters $\theta_{23}$ and $\Delta m_{31}^{2}$ have been established by the atmospheric neutrino data of SK [4]. Details of our reanalysis of the SK-I zenith angle distributed data can be found in Ref. 11] and references therein. The allowed region is shown in Fig. 1] (middle). 


\begin{tabular}{lcccc}
\hline \hline parameter & bf $\pm 1 \sigma$ & $1 \sigma$ acc. & $2 \sigma$ range & $3 \sigma$ range \\
\hline$\Delta m_{21}^{2}\left[10^{-5} \mathrm{eV}^{2}\right]$ & $7.9 \pm 0.3$ & $4 \%$ & $7.3-8.5$ & $7.1-8.9$ \\
$\left|\Delta m_{31}^{2}\right|\left[10^{-3} \mathrm{eV}^{2}\right]$ & $2.5_{-0.25}^{+0.20}$ & $10 \%$ & $2.1-3.0$ & $1.9-3.2$ \\
\hline $\sin ^{2} \theta_{12}$ & $0.30_{-0.03}^{+0.02}$ & $9 \%$ & $0.26-0.36$ & $0.24-0.40$ \\
$\sin ^{2} \theta_{23}$ & $0.50_{-0.07}^{+0.08}$ & $16 \%$ & $0.38-0.64$ & $0.34-0.68$ \\
$\sin ^{2} \theta_{13}$ & - & - & $\leq 0.025$ & $\leq 0.041$ \\
\hline \hline
\end{tabular}

TABLE I: Best fit values (bf), $1 \sigma$ errors, relative accuracies at $1 \sigma$, and $2 \sigma$ and $3 \sigma$ allowed ranges of three-flavor neutrino oscillation parameters from a combined analysis of global data.
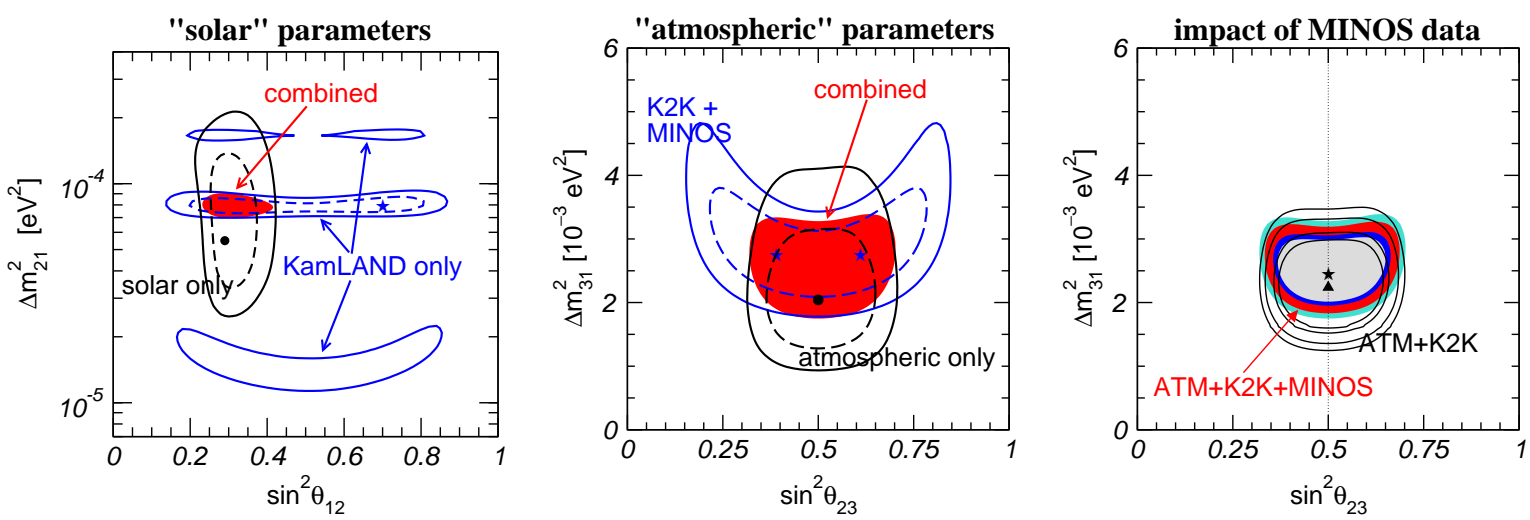

FIG. 1: Determination of the leading oscillation parameters from an interplay of experiments with natural and artificial neutrino sources (left and middle panels). In the right panel the allowed regions are shown with (colored regions) and without (contour curves) MINOS data. In the left and middle panels the allowed regions are shown at 90\% CL (dashed curves) and 99.73\% CL (solid curves and shaded regions), whereas in the right panel regions are shown at $90 \%, 95 \%, 99 \%$, and $99.73 \%$ CL.

Also In this case, by now we have an independent confirmation of the effect by experiments based on man-made neutrinos, namely the first generation of long-baseline (LBL) accelerator experiments exploring the $\nu_{\mu}$ disappearance oscillation channel. In the $\mathrm{K} 2 \mathrm{~K}$ experiment [8] the neutrino beam is produced at the KEK proton synchrotron, and originally consists of $98 \%$ muon neutrinos with a mean energy of $1.3 \mathrm{GeV}$. The $\nu_{\mu}$ content of the beam is observed at the SK detector at a distance of $250 \mathrm{~km}$. For the $\mathrm{K} 2 \mathrm{~K}-\mathrm{I}$ and $\mathrm{K} 2 \mathrm{~K}-\mathrm{II}$ data $\left(0.89 \times 10^{20}\right.$ p.o.t. in total $)$ 107 events have been detected, whereas $151_{-10}^{+12}$ have been expected for no oscillations.

Recently first data $\left(0.93 \times 10^{20}\right.$ p.o.t. $)$ from the MINOS experiment have been released [9]. A neutrino beam with $98.5 \%\left(\nu_{\mu}+\bar{\nu}_{\mu}\right)$ and a mean energy of $3 \mathrm{GeV}$ is produced at Fermilab and observed at the MINOS detector in the Soudan mine at a distance of $735 \mathrm{~km}$. In the absence of oscillations $177 \pm 11 \nu_{\mu}$ events with $E<10 \mathrm{GeV}$ are expected, whereas 92 have been observed, which provides a $5.0 \sigma$ evidence for disappearance. In our re-analysis we use spectral data divided into 15 bins in reconstructed neutrino energy, and our allowed region from MINOS-only is in very good agreement with the official result [9]. The values of the oscillation parameters from MINOS are consistent with the ones from $\mathrm{K} 2 \mathrm{~K}$, as well as from SK atmospheric data.
The impact of the data from MINOS in the global analysis is illustrated in Fig. 1 (right). We find that the best fit point for $\Delta m_{31}^{2}$ is shifted upward from $2.2 \times 10^{-3} \mathrm{eV}^{2}$ for SK+K2K to $2.5 \times 10^{-3} \mathrm{eV}^{2}$. In addition MINOS improves the lower bound on $\Delta m_{31}^{2}$, which is increased from $1.4 \times 10^{-3} \mathrm{eV}^{2}$ for $\mathrm{SK}+\mathrm{K} 2 \mathrm{~K}$ to $1.9 \times 10^{-3} \mathrm{eV}^{2}$ at $3 \sigma$. The relative accuracy on $\Delta m_{31}^{2}$ at $1 \sigma$ is improved from $14 \%$ to $10 \%$. As obvious from the middle panel of Fig. 1 the determination of $\theta_{23}$ is completely dominated by atmospheric data and there is no change due to MINOS. Let us add that present data cannot distinguish between $\Delta m_{31}^{2}>0$ and $<0$, and hence, both, the normal and inverted neutrino mass hierarchies provide equally good fits to the data.

\section{THE BOUND ON $\theta_{13}$}

Similar to the case of the leading oscillation parameters, also the bound on $\theta_{13}$ emerges from an interplay of different data sets, as we illustrate in Fig. 2. An important contribution to the bound comes, of course, from the $\mathrm{CHOOZ}$ reactor experiment combined with the determination of $\Delta m_{31}^{2}$ from atmospheric and LBL experiments. However, due to a complementarity of low and high energy solar data, as well as solar and KamLAND data also 


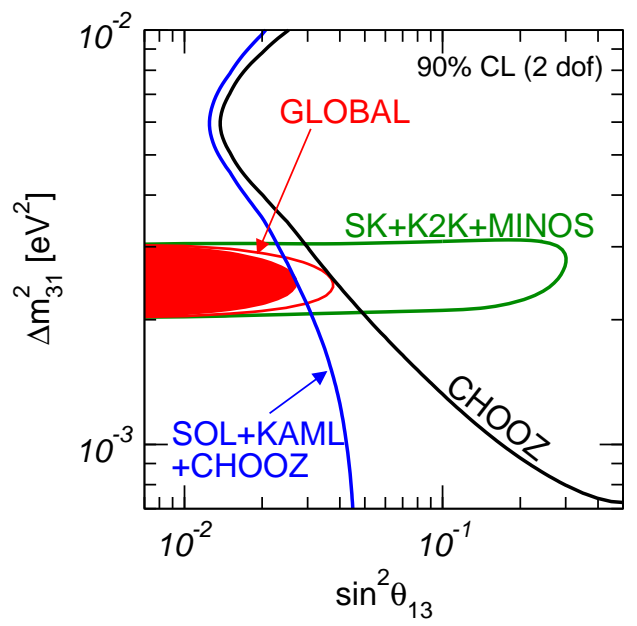

FIG. 2: The bound on $\sin ^{2} \theta_{13}$ from the interplay of the global data.

solar+KamLAND provide a non-trivial constraint on $\theta_{13}$, see e.g., Refs. 10, 11, 15]. We find at 90\% CL $(3 \sigma)$ the following limits:

$\sin ^{2} \theta_{13}< \begin{cases}0.027(0.058) & \text { CHOOZ+atm+LBL, } \\ 0.033(0.071) & \text { solar+KamLAND, } \\ 0.020(0.041) & \text { global data. }\end{cases}$

The addition of MINOS data leads to a slight tightening of the constraint (the $3 \sigma$ limit from $\mathrm{CHOOZ}+\mathrm{atm}+\mathrm{K} 2 \mathrm{~K}$ is shifted from 0.067 to 0.058 if MINOS is added) because of the stronger lower bound on $\Delta m_{31}^{2}$, where the CHOOZ bound becomes weaker (c.f. Fig. 22). Note that also the update in the solar model 13. leads to a small shift in the limit from solar+KamLAND data (from 0.079 to 0.071 at $3 \sigma)$. Both of these updates contribute to the change of the global bound from 0.046 [16] to 0.041 at $3 \sigma$.

\section{SUB-LEADING EFFECTS IN ATMOSPHERIC NEUTRINOS}

In principle one expects that at some level subleading effects will show up in atmospheric neutrinos, involving oscillations with $\Delta m_{21}^{2}$ or effects of a finite $\theta_{13}$, see e.g., Refs. 17, 18, 19, 20, 21]. An excess of $e$-like events observed in SK [ 5 ] might be a possible hint for such effects, and in Refs. [19, 20] a slight preference for non-maximal values of $\theta_{23}<$ $\pi / 4$ has been found. In contrast, the SK analysis presented in Ref. 21] did not confirm that hint.

From a full three-flavor analysis of SK data 22] shown in Fig. 3 one finds that indeed sub-GeV data prefer a value $\theta_{23}<\pi / 4$, however, if only multi-GeV data is used the best fit occurs for $\theta_{23}>\pi / 4$. Summing sub- and multi-GeV data leads incidentally to a cancellation of both effects and the best fit occurs very close to maximal mixing. Finally, using all

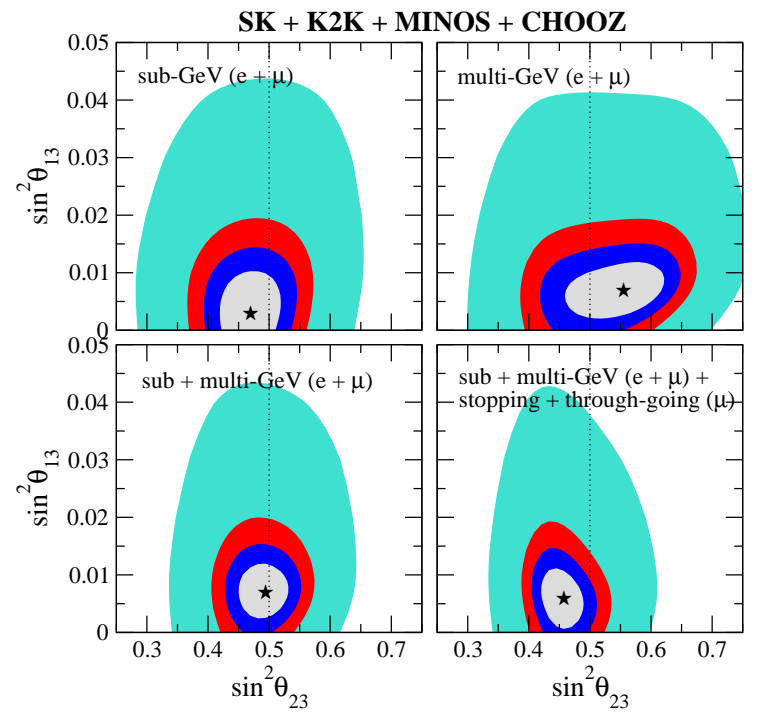

FIG. 3: Contours of $\Delta \chi^{2}=0.2,0.5,1,4.6$ in the plane $\sin ^{2} \theta_{23}-\sin ^{2} \theta_{13}$ from various SK data samples, taking into account oscillations with $\Delta m_{21}^{2}=8 \times 10^{-5} \mathrm{eV}^{2}$.

data including sub-GeV, multi-GeV, stopping and through-going $\mu$-like data, the best fit moves again to $\sin ^{2} \theta_{23}=0.46[19]$. From these considerations we conclude that the final result for $\theta_{23}$ appears as a delicate interplay of different data samples, involving cancellations of opposite trends. Hence the result is rather sensitive to the very fine details of the analysis. Let us stress that the $\Delta \chi^{2}$ contours shown in Fig. 3 correspond to $9.5 \%, 22 \%, 39 \%$, and $90 \%$ CL (2 d.o.f.), i.e., there is no significance in these effects. The purpose of this analysis is to show that present data does not allow to obtain statistically meaningful indications of non-maximal values of $\theta_{23}$ nor of non-zero values of $\theta_{13}$. Nevertheless, sub-leading three-flavor effects in atmospheric oscillations can be explored in future Mt scale water Čerenkov [23] or magnetized iron calorimeter 24] experiments, and may provide complementary information to LBL experiments.

Fig. 4 illustrates how details of the atmospheric neutrino analysis affect the bound on $\sin ^{2} \theta_{13}$ from $\mathrm{CHOOZ}+\mathrm{atm}+\mathrm{K} 2 \mathrm{~K}$ data. It is evident from the figure that the inclusion of three-flavor effects (from $\theta_{13}$ and/or $\Delta m_{21}^{2}$ ), as well as different treatments of systematics lead to an "uncertainty" of about $16 \%$ on the bound on $\sin ^{2} \theta_{13}$ at $2 \sigma$, as indicated by the "error bar" in the figure. Note that the shifts of the global $\theta_{13}$ limit due to MINOS or changes in the solar neutrino analysis reported in Sec. IIII are at the same level as this uncertainty from details in the atmospheric neutrino analysis. 


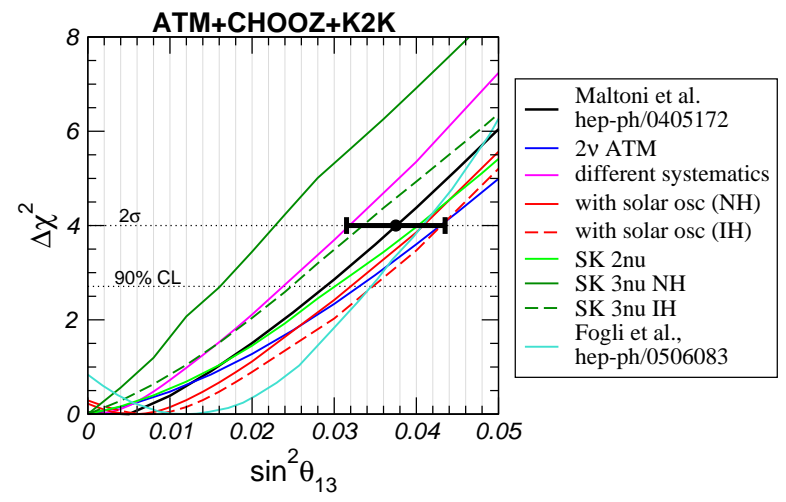

FIG. 4: Dependence of the bound on $\sin ^{2} \theta_{13}$ from $\mathrm{CHOOZ}+\mathrm{atm}+\mathrm{K} 2 \mathrm{~K}$ data on the details of the atmospheric neutrino analysis. The curves (from top to bottom in the legend) correspond to (i) the analysis of Ref. 11] without $\Delta m_{21}^{2}$-effects, (ii) a 2-flavor analysis neglecting $\theta_{13}$ effects in ATM, (iii) an analysis with a different systematical error treatment 25], (iv) taking into account the effect of $\Delta m_{21}^{2}$ for both types of the neutrino mass hierarchy, $(v)$ using the 2 -flavor SK analysis [5], (vi) the SK analysis [26] taking into account $\theta_{13}$ effects for both hierarchies, and (vii) the analysis from Ref. 20].

\section{THE LSND PROBLEM}

To reconcile the LSND evidence [12] for $\bar{\nu}_{\mu} \rightarrow \bar{\nu}_{e}$ oscillations with $\Delta m^{2} \sim \mathrm{eV}^{2}$ is a long-standing problem for neutrino phenomenology, and the community is eagerly waiting for an experimental answer to this problem from the MiniBooNE experiment 27]. The three required mass-squared differences can be obtained in four-neutrino mass schemes, but such models cannot accommodate the constraints on the mixing [28] (see Ref. 11] for an updated analysis): Mass schemes of the $(2+2)$ type predict that a large fraction of the sterile neutrino participates in solar and/or atmospheric neutrino oscillations, which in both cases is disfavored by the data 28, 29], and therefore such schemes are ruled out at more than $5 \sigma \mathrm{CL}$. The $(3+1)$ mass spectra are in perfect agreement with solar and atmospheric data, however, they suffer from a tension between the LSND signal and null-result short-baseline disappearance experiments 30, 31], most importantly Bugey [32] and CDHS [33], which disfavors these models at the $3 \sigma$ level.

In Ref. 34 a five-neutrino mass scheme of the type $(3+2)$ has been considered to avoid these constraints, and it is claimed that the disagreement measured by the so-called parameter goodness-offit [35] is improved from $0.032 \%$ for $(3+1)$ to $2.1 \%$. However, it should be noted that, apart from possible severe conflicts with constraints from cosmology, the best fit point found in Ref. 34] seems to be disfavored also from atmospheric neutrino data. As pointed out in Ref. 31] atmospheric neutrinos pro-

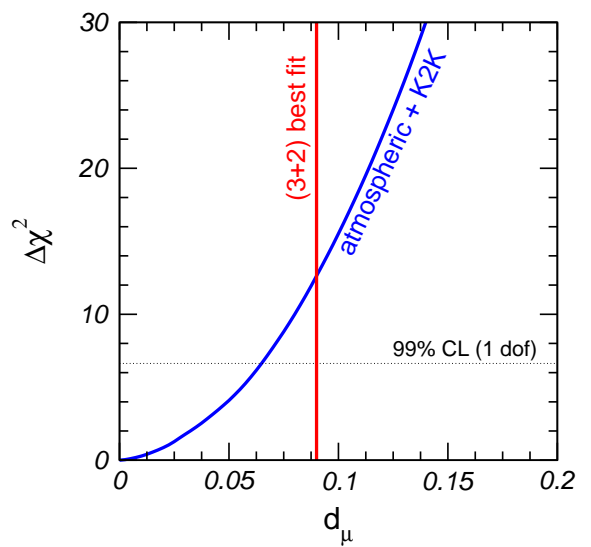

FIG. 5: Constraint on the parameter $d_{\mu}$ from atm $+\mathrm{K} 2 \mathrm{~K}$ data from Ref. 11] compared to the best fit prediction of the $(3+2)$ neutrino mass scheme [34].

vide a constraint on a parameter $d_{\mu}$, denoting the fraction of $\nu_{\mu}$ which does not participate in oscillations with $\Delta m_{\mathrm{atm}}^{2}$. In the $(3+2)$ scheme this parameter is given by $d_{\mu}=\left|U_{\mu 4}\right|^{2}+\left|U_{\mu 5}\right|^{2}$, and with the best fit values [34] $U_{\mu 4}=0.204, U_{\mu 5}=0.224$ one finds $d_{\mu} \approx 0.09$. As visible from Fig. [5 this value leads to a $\Delta \chi^{2} \approx 12.5$ from atmospheric $+\mathrm{K} 2 \mathrm{~K}$ data, and hence seems to be disfavored at the $3.5 \sigma$ level. Therefore, a re-analysis of the $(3+2)$ scenario including the constraint from atmospheric data seems to be required to judge the viability of this model.

In view of these difficulties to explain the LSND result with neutrino oscillations several alternative mechanisms have been proposed, see Ref. 36] for references. In addition to the fact that some of them involve very speculative physics, many of these proposals have also phenomenological problems to accommodate all constraints. Scenarios which seem to be in agreement with all present data are a model with a decaying sterile neutrino 36 , a four-neutrino mass scheme plus CPT violation [37], and a model based on sterile neutrinos and large extra dimensions 38].

\section{SUMMARY}

In this talk I have summarized the status of neutrino oscillations with May 2006, providing updated best fit values and allowed ranges of the three-flavor neutrino oscillation parameters. The impact of the recently released first data from MINOS on the determination of $\Delta m_{31}^{2}$ as well as on the bound on $\theta_{13}$ has been investigated. Furthermore, sub-leading effects in atmospheric neutrino data have been discussed, stressing that hints for non-maximal values of $\theta_{23}$ and/or non-zero values of $\theta_{13}$ depend on the fine details of the analysis and are not statistically significant. In view of the upcoming results from 
MiniBooNE I have reviewed once again the problem related to the LSND result, and a confirmation of the effect by MiniBooNE would imply a serious challenge to neutrino oscillation phenomenology.

Acknowledgments. I thank the organizers for the very pleasant and stimulating workshop. The results presented here have been obtained in col- laboration with M. Maltoni, M.A. Tórtola, and J.W.F. Valle, and in particular I would like to thank M. Maltoni for the permission to use his atmospheric neutrino code for the analysis of sub-leading effects. T.S. is supported by a "Marie Curie IntraEuropean Fellowship within the 6th European Community Framework Program."
[1] B. T. Cleveland et al., Astrophys. J. 496 (1998) 505; J.N. Abdurashitov et al. (SAGE), J. Exp. Theor. Phys. 95 (2002) 181 astro-ph/0204245; T. Kirsten et al. (GALLEX and GNO), Nucl. Phys. B (Proc. Suppl.) 118 (2003) 33; C. Cattadori, Talk given at Neutrino04, June 14-19, 2004, Paris, France; S. Fukuda et al. (Super-K), Phys. Lett. B539 (2002) 179.

[2] Q.R. Ahmad et al. (SNO), Phys. Rev. Lett. 89, 011302 (2002) nucl-ex/0204009.

[3] B. Aharmim et al. (SNO), Phys. Rev. C 72, 055502 (2005) nucl-ex/0502021.

[4] Y. Fukuda et al. (Super-K), Phys. Rev. Lett. 81 (1998) 1562 hep-ex/9807003;

[5] Y. Ashie et al. (Super-K), Phys. Rev. D 71 (2005) 112005 hep-ex/0501064.

[6] T. Araki et al. (KamLAND), Phys. Rev. Lett. 94, 081801 (2005) hep-ex/0406035.

[7] M. Apollonio et al. (CHOOZ), Eur. Phys. J. C 27, 331 (2003) hep-ex/0301017.

[8] E. Aliu et al. (K2K), Phys. Rev. Lett. 94, 081802 (2005) hep-ex/0411038.

[9] E. Ables et al. (MINOS) FERMILAB-PROPOSALP-875; E. Falk Harris, these proceedings; http://www-numi.fnal.gov/talks/results06.html

[10] M. Maltoni, T. Schwetz, M.A. Tórtola and J.W.F. Valle, Phys. Rev. D 68, 113010 (2003) hep-ph/0309130.

[11] M. Maltoni, T. Schwetz, M.A. Tórtola and J.W.F. Valle, New J. Phys. 6 (2004) 122 hep-ph/0405172.

[12] A. Aguilar et al. (LSND), Phys. Rev. D 64, 112007 (2001) hep-ex/0104049.

[13] J. N. Bahcall, A. M. Serenelli and S. Basu, Astrophys. J. 621, L85 (2005) astro-ph/0412440.

[14] P. Huber and T. Schwetz, Phys. Rev. D 70 (2004) 053011 hep-ph/0407026.

[15] S. Goswami and A.Y. Smirnov, Phys. Rev. D 72, 053011 (2005) hep-ph/0411359.

[16] T. Schwetz, Acta Phys. Polon. B 36 (2005) 3203 hep-ph/0510331.

[17] E.K. Akhmedov et al., Nucl. Phys. B 542 (1999) 3; hep-ph/9808270; J. Bernabeu, S. Palomares Ruiz, S.T. Petcov, Nucl. Phys. B 669 (2003) 255. hep-ph/0305152.

[18] C. W. Kim and U. W. Lee, Phys. Lett. B 444 (1998) 204 hep-ph/9809491; O.L.G. Peres, A.Y. Smirnov, Nucl. Phys. B 680 (2004) 479. hep-ph/0309312.
[19] M.C. Gonzalez-Garcia, M. Maltoni and A.Y. Smirnov, Phys. Rev. D 70 (2004) 093005 hep-ph/0408170.

[20] G. L. Fogli, E. Lisi, A. Marrone and A. Palazzo, hep-ph/0506083

[21] Talk by T.Kajita at NuFact05, June 21-26, Frascati, Italy, http://www.lnf.infn.it/conference/nufact05/

[22] M. Maltoni, private communication.

[23] P. Huber, M. Maltoni, T. Schwetz, Phys. Rev. D 71 (2005) 053006 hep-ph/0501037; M. Maltoni, these proceedings.

[24] S. T. Petcov and T. Schwetz, Nucl. Phys. B 740 (2006) 1 hep-ph/0511277; S. Goswami, these proceedings.

[25] M. C. Gonzalez-Garcia and M. Maltoni, Phys. Rev. D 70 (2004) 033010 hep-ph/0404085.

[26] J. Hosaka et al. (Super-K), hep-ex/0604011

[27] J. Monroe (MiniBooNE), hep-ex/0406048

[28] M. Maltoni, T. Schwetz, M. A. Tortola and J. W. F. Valle, Nucl. Phys. B 643 (2002) 321 hep-ph/0207157.

[29] M. C. Gonzalez-Garcia, M. Maltoni and C. Pena-Garay, Phys. Rev. D 64 (2001) 093001 hep-ph/0105269.

[30] S. M. Bilenky, C. Giunti and W. Grimus, Eur. Phys. J. C 1 (1998) 247 hep-ph/9607372; N. Okada and O. Yasuda, Int. J. Mod. Phys. A 12, 3669 (1997) hep-ph/9606411; V. D. Barger, S. Pakvasa, T. J. Weiler and K. Whisnant, Phys. Rev. D 58 (1998) 093016 hep-ph/9806328; W. Grimus and T. Schwetz, Eur. Phys. J. C 20 (2001) 1 hep-ph/0102252.

[31] S. M. Bilenky, C. Giunti, W. Grimus and T. Schwetz, Phys. Rev. D 60 (1999) 073007 hep-ph/9903454.

[32] Y. Declais et al., Nucl. Phys. B 434, 503 (1995).

[33] F. Dydak et al., Phys. Lett. B 134, 281 (1984).

[34] M. Sorel, J. M. Conrad and M. Shaevitz, Phys. Rev. D 70, 073004 (2004) hep-ph/0305255.

[35] M. Maltoni and T. Schwetz, Phys. Rev. D 68, 033020 (2003) hep-ph/0304176.

[36] S. Palomares-Ruiz, S. Pascoli and T. Schwetz, JHEP 0509 (2005) 048 hep-ph/0505216.

[37] V. Barger, D. Marfatia and K. Whisnant, Phys. Lett. B 576, 303 (2003) hep-ph/0308299.

[38] H. Pas, S. Pakvasa and T. J. Weiler, Phys. Rev. D 72, 095017 (2005) hep-ph/0504096. 